\title{
A assistência do enfermeiro no pré-natal
}

\author{
Nurse assistance in prenatal care \\ Asistencia de enfermería en la atención prenatal
}

Recebido: 22/10/2021 | Revisado: 27/10/2021 | Aceito: 29/10/2021 | Publicado: 01/11/2021

Ludymilla de Oliveira Menezes

ORCID: https://orcid.org/0000-0002-7826-9224

Faculdade Integrada Carajás, Brasil

E-mail: menezes.ludymilla14@ gmail.com

Nayane da Silva Almeida

ORCID: https://orcid.org/0000-0003-3782-5068

Faculdade Integrada Carajás, Brasil

E-mail: nayane.almeidafda55@gmail.com

Marcos Vinícios Ferreira dos Santos

ORCID: https://orcid.org/0000-0003-1335-1021

Faculdade Integrada Carajás, Brasil

E-mail: viniciosferreirasantos@ hotmail.com

\begin{abstract}
Resumo
O pré-natal realizado pelo enfermeiro é importante, para a prevenção ou detecção precoce de alterações patológicas, proporcionando assim uma gestação saudável e com um bom desenvolvimento do feto. Objetivo: O estudo teve como objetivo conhecer, entender e verificar como se dar a assistência ao pré-natal realizada pelo enfermeiro na atenção básica. Metodologia: Trata-se de uma revisão integrativa narrativa de literatura, que buscou publicações cientificas de 2014 a 2021, nas plataformas online BVS, ScIELO BRASIL e GOOGLE ACADÊMICO. Utilizando as seguintes palavras chaves: "Gravidez", "Pré-natal", "Assistência do enfermeiro". Utilizado os termos: "Pré-natal", "assistência do enfermeiro". Resultados: Os resultados encontrados foram, que a assistência no pré-natal acolhe a mulher desde o início da gravidez, assegurando no fim da gestação, o nascimento de uma criança saudável e a garantia do bem-estar materno e neonatal, promovendo a saúde e identificando, antecipadamente surgimento de anomalias, e reduzindo a mortalidade neonatal. Considerações finais: O processo gestacional e puerperal traz alterações para o corpo tanto fisiologicamente quanto o emocional da mulher, além da mudança na rotina da família. O profissional enfermeiro que acompanha essa gestante, desde o pré-natal, pode atuar de forma a reduzir anormalidades que possam interferir no processo gestacional.
\end{abstract}

Palavras-chave: Gravidez; Pré-natal; Assistência do enfermeiro.

\begin{abstract}
Prenatal care performed by nurses is important for the prevention or early detection of pathological changes, thus providing a healthy pregnancy and a good development of the fetus. Objective: The study aimed to know, understand and verify how to provide prenatal care provided by nurses in primary care. Methodology: This is an integrative narrative literature review, which sought scientific publications from 2014 to 2021, on the online platforms BVS, ScIELO BRASIL and GOOGLE ACADÊMICO. Using the following key words: "Pregnancy", "Prenatal", "Nurse assistance". Used the terms: "Prenatal", "nurse assistance". Results: The results found were that prenatal care welcomes the woman from the beginning of pregnancy, ensuring at the end of pregnancy, the birth of a healthy child and the guarantee of maternal and neonatal well-being, promoting health and identifying, early onset of anomalies, and reducing neonatal mortality. Final considerations: The gestational and puerperal process brings changes to the woman's body, both physiologically and emotionally, in addition to the change in the family's routine. The professional nurse who accompanies this pregnant woman, from the prenatal period, can act to reduce abnormalities that may interfere with the gestational process.
\end{abstract}

Keywords: Pregnancy; Prenatal; Nurse assistance.

\section{Resumen}

La atención prenatal realizada por enfermeras es importante para la prevención o detección temprana de cambios patológicos, proporcionando así un embarazo saludable y un buen desarrollo del feto. Objetivo: El estudio tuvo como objetivo conocer, comprender y verificar cómo se brinda la atención prenatal brindada por enfermeras en atención primaria. Metodología: Se trata de una revisión de literatura narrativa integradora, que buscó publicaciones científicas de 2014 a 2021, en las plataformas online BVS, ScIELO BRASIL y GOOGLE ACADÊMICO. Utilizando las siguientes palabras clave: "Embarazo", "Prenatal", "Asistencia de enfermería". Usó los términos: "Prenatal", "asistencia de enfermería". Resultados: Los resultados encontrados fueron que la atención prenatal acoge a la mujer desde el inicio del embarazo, asegurando al final del embarazo, el nacimiento de un niño sano y la garantía del 
bienestar materno y neonatal, promoviendo la salud e identificando, inicio temprano de la gestación. anomalías y reducción de la mortalidad neonatal. Consideraciones finales: El proceso gestacional y puerperal trae cambios en el cuerpo de la mujer, tanto fisiológica como emocionalmente, además del cambio en la rutina familiar. La enfermera profesional que acompaña a esta gestante, desde el período prenatal, puede actuar para reducir anomalías que puedan interferir con el proceso gestacional.

Palabras clave: embarazo; Prenatal; Asistencia de enfermería.

\section{Introdução}

O pré-natal realizado pelo enfermeiro é importante, para a prevenção ou detecção precoce de alterações patológicas, proporcionando assim uma gestação saudável e com um bom desenvolvimento fetal. $\mathrm{O}$ vínculo que o enfermeiro deve ter com a paciente também é fundamental para esse que processo seja realizado de forma adequada (Viellas, et al., 2014).

Realizando atividades instrutivas no período gestacional, parto e puerpério compete ao enfermeiro acompanhar as mulheres de baixo risco e sua família. E compete ao enfermeiro realizar as consultas, solicitação de exames, orientação, registrar informações no prontuário e na caderneta da gestante em cada consulta, encaminhar de acordo com a sua necessidade, promover atividades educativas e incluir a participação de seus familiares, coletar colpo citológico e realiza visitas domiciliares (Pereira, et al., 2018).

O presente trabalho torna-se relevante e se justifica em razão, do pré-natal ser uma rotina do serviço de enfermagem dentro da atenção básica, e a assistência ao pré-natal de qualidade o acompanhamento ao processo de desenvolvimento da gestação e a educação a saúde na gestação realizada pelo enfermeiro é de extrema importância, pois, muitas vezes o único vínculo de confiança e conhecimento é com o enfermeiro, portanto se faz necessário conhecer a atuação do mesmo no acompanhamento do pré-natal. Substituindo conceitos errôneos por novos ensinamentos (Viellas, et al., 2014).

Para guiar o presente estudo, elaborou-se a seguinte questão norteadora: nos artigos analisados, qual a importância do enfermeiro na assistência ao pré-natal?

\section{Metodologia}

Este estudo é caracteriza-se como uma revisão integrativa narrativa da literatura que é utilizado de mapeamentos de estudos originais que são publicados ser apreciados temas que, incluem estudos qualitativos, quantitativos e teóricos com a finalidade de sintetizar segmentos da pesquisa em relação ao tema em questão. (Sousa, et al., 2018)

A busca foi realizada abrangendo as produções científicas eletrônicas produzidas entre 2014 a 2021, indexados nas bases de dados eletrônicos da Biblioteca Virtual em Saúde (BVS), Google acadêmico e Scielo - Brasil. Optou-se pela Google acadêmico, Scielo e Biblioteca Virtual em Saúde BVS por conter várias bases de dados, consequentemente um amplo número de artigos relacionado ao assunto abordado e, objetivando obter os resultados fidedignos da realidade atual dos cuidados de enfermagem no período do pré-natal.

Como critérios de inclusão, foram utilizados os seguintes filtros: texto completo disponível e gratuito que fossem artigos em idioma português, publicado nos anos de 2014 - 2021. Primeiro foi realizada leitura exploratória dos títulos e dos resumos dos artigos a fim de reconhecer os artigos que respondessem à pergunta norteadora. Em seguida foi realizada a escolha do material conforme a proposta do estudo e, por fim foi realizada a leitura interpretativa.

\section{Resultados e Discussão}

A (Figura 1) é uma representação gráfica das seleções dos artigos no trajeto deste trabalho, onde foram coletados dados através dos artigos analisados e selecionados para este estudo, segundo os critérios de inclusão. 
Figura 1. Fluxograma da seleção dos artigos.

\section{Verificações dos artigos originais nos bancos de dados}

Utilizando-se estes Descritos: Gravidez;

Pré-natal; Assistência do Enfermeiro.

Critérios de Inclusão: artigos originais, textos completos, recorte temporal 2014 a 2021 nas bases de dados BVS, ScIELO e GOOGLE ACADÊMICO

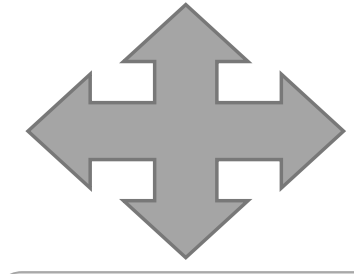

Leitura dos títulos resumos para selecionar 262 artigos

Fonte: Autores (2021).

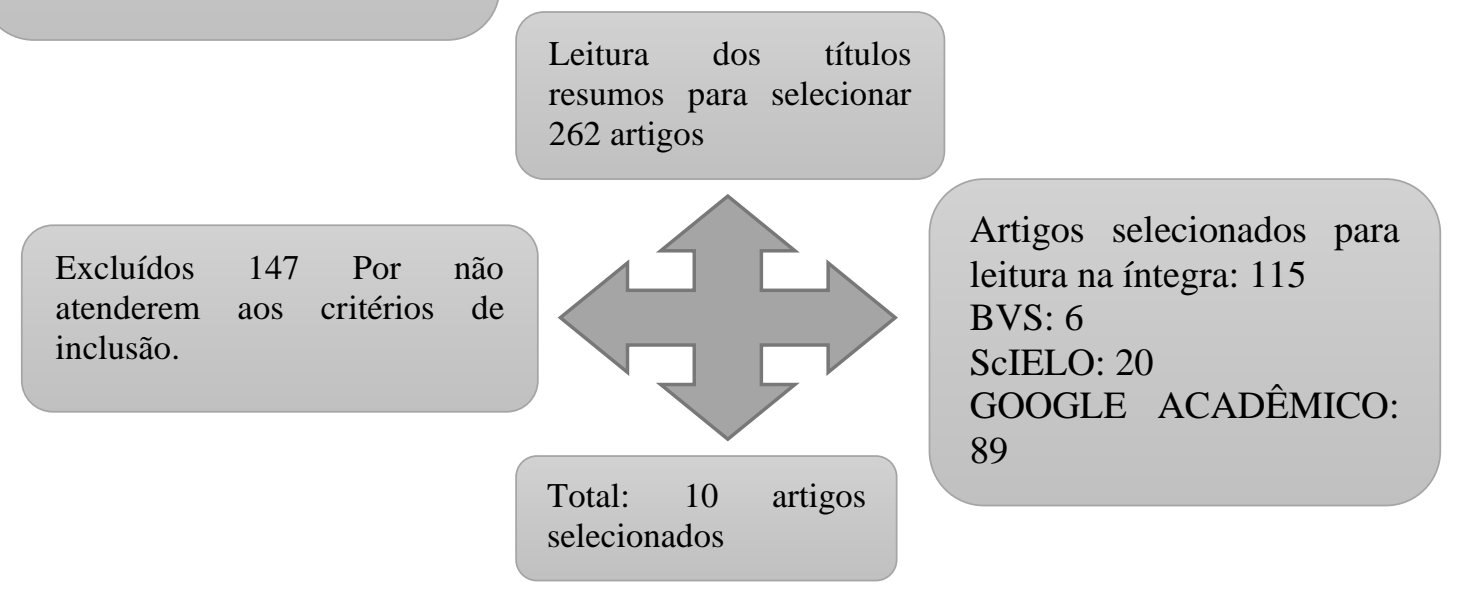

Referências encontradas:

BVS: 24

ScIELO: 38

GOOGLE ACADÊMICO: 200

Total: 262

A assistência do enfermeiro no pré-natal tem sido abordada de forma frequente na literatura. (Quadro 1) apresenta-se os artigos selecionados sendo relacionados os títulos, autores, ano de publicação e o principal ponto a ser discutido, a importância da assistência do enfermeiro no pré-natal. Assim possibilitando uma visão geral dos artigos que foram relacionados para o embasamento deste estudo. 
Quadro 1- Apresentação dos artigos, out/2021.

\begin{tabular}{|c|c|c|c|}
\hline Artigo & Autores & $\begin{array}{c}\text { Ano de } \\
\text { publicação }\end{array}$ & $\begin{array}{l}\text { A importância da assistência do } \\
\text { enfermeiro no pré-natal }\end{array}$ \\
\hline $\begin{array}{l}\text { 1.Percepção das gestantes quanto a } \\
\text { importância das ações educativas } \\
\text { promovida pelo enfermeiro no pré- } \\
\text { natal em uma unidade básica de } \\
\text { saúde. }\end{array}$ & Dias, E. G., et al. & 2015 & $\begin{array}{l}\text { Um dos principais objetivos na atenção ao pré- } \\
\text { natal e puerperal é "acolher a mulher desde o } \\
\text { início da gravidez, assegurando no fim da } \\
\text { gestação, o nascimento de uma criança } \\
\text { saudável e a garantia do bem-estar materno e } \\
\text { neonatal". }\end{array}$ \\
\hline $\begin{array}{l}\text { 2.Percepções de puérperas sobre o } \\
\text { cuidado de enfermagem na } \\
\text { articulação alojamento }\end{array}$ & Strefling, I. S., et al. & 2017 & $\begin{array}{l}\text { Traz um papel importante reduzindo a } \\
\text { mortalidade materna. }\end{array}$ \\
\hline $\begin{array}{l}\text { 3.Percepção de adolescentes } \\
\text { gestantes sobre a assistência de } \\
\text { enfermagem ao pré-natal. }\end{array}$ & $\begin{array}{l}\text { Carvalho, S. S. \& } \\
\text { Oliveira L. F. }\end{array}$ & 2020 & $\begin{array}{l}\text { É fundamental para com o cuidado da saúde da } \\
\text { mulher e de seu bebê, desde a identificação da } \\
\text { gravidez precoce, até o momento do parto. }\end{array}$ \\
\hline $\begin{array}{l}\text { 4.Promoção do aleitamento materno } \\
\text { na assistência pré-natal pelo } \\
\text { enfermeiro. }\end{array}$ & Sardinha, D. M., et al. & 2019 & $\begin{array}{l}\text { Promovendo a saúde e identificando, } \\
\text { antecipadamente, problemas que possam } \\
\text { resultar em risco para a saúde da gestante e do } \\
\text { concepto. }\end{array}$ \\
\hline $\begin{array}{l}\text { 5.Aplicação da sistematização da } \\
\text { Assistência de enfermagem em } \\
\text { gestantes atendidas no pré-natal. }\end{array}$ & Silva, J. C. B., et al. & 2019 & $\begin{array}{l}\text { O acompanhamento do pré-natal visa assegurar } \\
\text { o desenvolvimento, favorecendo um } \\
\text { nascimento saudável abordando atividades } \\
\text { psicossociais e atividades preventivas }\end{array}$ \\
\hline $\begin{array}{l}\text { 6.Fatores evitáveis para mortalidade } \\
\text { neonatal: uma revisão narrativa da } \\
\text { literatura. }\end{array}$ & Buges, N. M. & 2020 & $\begin{array}{l}\text { A maioria dos óbitos é considerada evitável } \\
\text { podendo ser prevenida com a melhoria da } \\
\text { assistência no pré-natal, parto e ao recém- } \\
\text { nascido. }\end{array}$ \\
\hline $\begin{array}{l}\text { 7.Rede Mãe Paranaense: assistência } \\
\text { ao pré-natal entre mulheres nos } \\
\text { extremos de idade }\end{array}$ & Souza, et al. & 2021 & $\begin{array}{l}\text { A inserção precoce da gestante no atendimento } \\
\text { pré-natal, tem como intuito oferecer garantia de } \\
\text { um atendimento no ambulatório para pacientes } \\
\text { de risco, garantia de parto hospitalar conforme a } \\
\text { classificação do risco gestacional. }\end{array}$ \\
\hline $\begin{array}{l}\text { 8.Ações do enfermeiro no pré-natal e } \\
\text { a importância atribuída pelas } \\
\text { gestantes. }\end{array}$ & Dias, E. G., et al. & 2018 & $\begin{array}{l}\text { A ação prestada pelo enfermeiro é importante no } \\
\text { pré-natal, sendo que por meio da assistência } \\
\text { prestada possibilitando a identificação de } \\
\text { alterações precoces e o monitoramento da } \\
\text { gestante. }\end{array}$ \\
\hline $\begin{array}{l}\text { 9.Consulta de enfermagem pré-natal: } \\
\text { narrativas de mulheres grávidas e } \\
\text { enfermeiras }\end{array}$ & Gomes, C. B., et al. & 2019 & $\begin{array}{l}\text { Intervindo com estratégias de promoção da } \\
\text { saúde, prevenção de doenças e prestar cuidados } \\
\text { humanizados para que a mãe e o bebê tenham } \\
\text { um cuidado saudável e um bom pré-natal. }\end{array}$ \\
\hline $\begin{array}{l}\text { 10.Qualidade de consulta de } \\
\text { enfermagem na assistência ao pré- } \\
\text { natal de risco habitual. }\end{array}$ & Oliveira, I. G., et al. & 2017 & $\begin{array}{l}\text { O papel do enfermeiro com suas ações } \\
\text { educativas é necessário na redução da } \\
\text { mortalidade materna, possuindo qualificação e } \\
\text { conhecimento para se ter um pré-natal de } \\
\text { qualidade e satisfatório. }\end{array}$ \\
\hline
\end{tabular}


Dois tópicos importantes são percebidos nestes artigos: No primeiro sobre a relevância do pré-natal para com a saúde da mãe e do bebe. No segundo a atuação do enfermeiro na assistência. Por isso para se obter uma boa discussão foram escolhidos estes dois tópicos.

\subsection{A importância do pré-natal}

É um sistema que tem a assistência de acolher, escutar e orientar para preparar e vivenciar a gestação tranquila e com saúde, consolidando papeis importantes como o pré-natal iniciando-se o mais cedo possível, apoiando a gestante emocionalmente, garantindo qualidade no desenvolvimento fetal e evitando óbitos fetais. (Dias, et al., 2015)

O pré-natal tem sua relevância no cuidado com a saúde da gestante e do seu bebê, possibilitando a identificação de uma gravidez antecipadamente. No cuidado com as orientações sobre sua gravidez que deve tomar. Cabendo ao enfermeiro sanar qualquer dúvida existente a ela. (Carvalho, S. S. \& De Oliveira, L. F., 2020)

Um pré-natal de qualidade assegura o desenvolvimento saudável sem risco para a saúde da mãe e para o bebe. A saúde deles é muito importante para o seu relacionamento de ambos pois é nesse momento que o enfermeiro a partir do prénatal evita tais intercorrências e os índices altos de morbimortalidade, materna e fetal. (Silva, J. C. B. et al., 2019)

Na realização do pré-natal é feita intervenções para melhorar a qualidade assistencial ao RN, com a finalidade de diminuir os índices de mortalidade neonatal, sendo os óbitos evitáveis, executando assim ações que serão redirecionadas para uma melhor qualidade, na assistência nos níveis de atenção à saúde. (Buges, N. M., 2020)

O cuidado feito no pré-natal assegura a mulher um parto seguro minimizando precocemente alterações que podem interferir nesse processo, como: a idade avançada, menor de idade, propensas a hipertensão, diabetes, obesidade, préeclâmpsia, parto tardio, espontâneo e cesáreo. (Souza, et al., 2021)

\subsection{A atuação do enfermeiro na assistência}

O enfermeiro ajuda na promoção da saúde identificando riscos na gestação. Onde cabe a ele realizar um pré-natal de qualidade consultas de baixo risco, solicitando exames de rotina, realização de exames obstétricos, solicita busca ativa, encaminhamentos se necessário, orientação do parto, cuidados com recém-nascido (RN), vacinação da gestante e com o RN, o aleitamento materno, e o afeto entre a mãe e o bebe. (Sardinha, et al., 2019)

O profissional de enfermagem tem a função de fazer orientações para as gestantes nas consultas tirando as dúvidas, orientando a mulher sobre a importância de cada consulta feita, os exames que são necessários durante a gestação, onde ele tem que ter um embasamento teórico e eficaz para auxilia-la do qual ela não tome atitudes de negligencia e imprudência. (Dias, E. G. et al., 2018)

O Ministério da Saúde (MS) recomenda que o enfermeiro dê informações e orientações importantes com intuito de minimizar possíveis alterações na gravidez e no puerpério evitando mudanças que possam ocorrer tanto psicológicas, físicas e sociais que essas gestantes e família iram vivenciar de forma harmônica e feliz. (Strefling, et al., 2017)

Para promover uma assistência de pré-natal de qualidade e atuar com estratégias na promoção da saúde, prevenindo as doenças como também na elaboração de um plano assistencial de consultas, humanização nos cuidados prestados, nas consultas de pré-natal. (Gomes, C. B. et al., 2019)

A assistência de enfermagem tem um papel importante acolhendo essa gestante e fazendo a anamnese, orientando e fazendo também exame físico, para que ela possa ter um pré-natal de qualidade e saudável, promovendo um parto sem intercorrências para a mãe e o bebe. (Oliveira, I. G. et al., 2017) 


\section{Considerações Finais}

O processo gestacional e puerperal traz alterações para o corpo tanto fisiologicamente quanto o emocional da mulher, além da mudança na rotina da família. O profissional enfermeiro que acompanha essa gestante, desde o pré-natal, pode atuar de forma a reduzir anormalidades que possam interferir no processo gestacional. O trabalho tem como intuito importante, de ressaltar como se dá a assistência ao pré-natal realizada pelo enfermeiro na atenção básica, conhecendo-a e entende-la. $\mathrm{O}$ enfermeiro tem um papel importante na assistência onde ele faz o acolhimento e tratamento da gestante, visando a humanização no tratamento do bebe e da mãe, assim trazendo os sentimentos emocionais, favorecendo a confiança no enfermeiro. O pré-natal é uma medida importante para a promoção e prevenção na saúde da gestante e do bebe, proporcionando uma melhor qualidade na vida e reduzindo os riscos à saúde. Torna-se o momento onde são tomadas as ações para promover a qualidade da saúde, através da realização procedimentos, exames, consultas, ações educativas em saúde envolvendo características da saúde da mulher no seu ciclo gravídico. Depois de analisados os artigos foi observado que o acompanhamento e a assistência prestada pelo enfermeiro no pré-natal, são de suma importância para que se realize um prénatal de qualidade, podendo evitar transtornos nessas fases, evitando a óbito fetal e mortalidade neonatal. O cuidado de enfermagem é toda ação realizada pelo enfermeiro durante sua prática, centrada no ser humano doente ou sadio, com suas necessidades biopsicossociais e espirituais, com atitudes de preocupação, de responsabilização e de envolvimento afetivo com o outro. Seu objetivo é centrado na promoção da saúde, na prevenção de doenças e na recuperação e reabilitação da saúde.

Almeja - se que este trabalho sirva de base para novos estudos, para se enriquecer com mais conhecimento, condutas e ações que possibilitam um pré-natal de qualidade, garantindo a saúde e o bem-estar materno e fetal, sem que aja intercorrências e dificuldades.

\section{Referências}

Andrade, H. S. (2017). Assistência do enfermeiro ao recém-nascido na atenção primária de saúde. Cadernos da Escola de Saúde. 17(2), 61-78.

Araújo, Â. C. L. D. \& Campos, R. R. (2020). Rede de atenção: saúde da mulher. 7 - 105.

Balsells, M. M. D., Oliveira, T. M. F. D., Bernardo, E. B. R., Aquino, P. D. S., Damasceno, A. K. D. C., Castro, R. C. M. B. \& Pinheiro, A. K. B. (2018). Evaluación del proceso en la atención prenatal de embarazadas con riesgo normal. Acta Paulista de Enfermagem, 31(3), $247-254$.

Barbosa, E. A. \& Fukusato, P. C. S. (2020). Manual Prático do Desenvolvimento Infantil. Thieme Revinter.

Batista, L. \& Matumoto, S. (2019). Validação de roteiro de sistematização da assistência de enfermagem à gestante na atenção primária. Revista Eletrônica Acervo Científico. $1-8$.

Brasil, M. S. (2014). Humanização do parto e do nascimento-Cadernos Humaniza SUS. Universidade Estadual do Ceará. Brasília. Ministério da Saúde, 4, 465 .

Buges, N. M. (2020). Fatores evitáveis para mortalidade neonatal: uma revisão narrativa da literatura. Amazônia: science \& health, 8(1), 2-14.

Campos, M. T. A.; Tilio, R. \& Crema, I. L. (2017). Socialização, gênero e família: uma revisão integrativa da literatura científica. Pensando famílias. 21 (1), $146-161$.

Carpes, F., Biff, D., \& Stumm, K. E. (2016). Percepção de acadêmicos de enfermagem acerca do papel do enfermeiro no cuidado pré-natal. Revista Enfermagem Atual In Derme, 79(17).

Carvalho, S. S., \& de Oliveira, L. F. (2020). Percepção de adolescentes gestantes sobre a assistência de enfermagem ao pré-natal. Enfermagem em Foco, 11(3).

Oliveira, E. C., de Meira Barbosa, S., \& Melo, S. E. P. (2016). A importância do acompanhamento pré-natal realizado por enfermeiros. Revista Científica FacMais, 7(3).

Sousa, L. M. M., Firmino, C. F., Marques-Vieira, C. M. A., Severino, S. S. P., \& Pestana, H. C. F. C. (2018). Revisões da literatura científica: tipos, métodos e aplicações em enfermagem. Revista Portuguesa de Enfermagem de Reabilitação, 1(1), 45-54.

Dias, E. G., dos Anjos, G. B., Alves, L., Pereira, S. N., \& Campos, L. M. (2018). Ações do enfermeiro no pré-natal e a importância atribuída pelas gestantes. Revista Sustinere, 6(1), 52-62.

Dias, E. G., do Espírito Santo, F. G., dos Santos, I. G. R., Alves, J. C. S., \& dos Santos, T. M. F. (2015). Percepção das gestantes quanto à importância das ações educativas promovida pelo enfermeiro no pré-natal em uma unidade básica de saúde. Revista Eletrônica Gestão e Saúde, (3), $2695-2710$. 
do Nascimento, I. B., Fleig, R., Pacheco, V. C., de Souza, M. L. R., Pinheiro, E. B., Ribeiro, T., \& Silva, J. C. (2017). Assistência pré-natal e resultado perinatal. Revista Brasileira em Promoção da Saúde, 30(2).

Duarte, S. J. H., \& de Almeida, E. P. (2014). O papel do enfermeiro do programa saúde da família no atendimento pré-natal. Revista de Enfermagem do Centro-Oeste Mineiro.

Fernandes, R. Z. S., \& Vilela, M. F. D. G. (2014). Estratégias de integração das práticas assistenciais de saúde e de vigilância sanitária no contexto de implementação da Rede Cegonha. Ciência \& Saúde Coletiva, 19, 4457-4466.

Fernandes, R. F. M., Meincke, S. M. K., Thumé, E., Soares, M. C., Collet, N., \& Carraro, T. E. (2015). Características do pré-natal de adolescentes em capitais das regiões Sul e Nordeste do Brasil1. Texto \& Contexto-Enfermagem, 24, 80-86.

Gomes, C. B., Dias, R. S., Silva, W. G., Pacheco, M. A., Sousa, F. G., \& Loyola, C. M. (2019). Prenatal nursing consultation: Narratives of pregnant women and nurses. Texto \& Contexto Enfermagem.

Guimarães, P. D. R. F., da Silva, A. N., de Rodrigues, C. R., Taneda, M., \& Zaniolo, L. M. (2015). O papel do enfermeiro no pré-natal humanizado realizado no psf. Revista da Saúde da AJES, 1(2).

Jerke, L. C., Oliveira, G., Sehnem, G. D., \& Schimith, M. D. (2019). A consulta de pré-natal de enfermagem em campo prático: relato de experiência nursing prenatal consultation in a practical field: experience report. II Seminário de Atenção Multiprofissional à Saúde do Neonato, Criança Adolescente e Família, 37.

Luz, L. A. D., Aquino, R., \& Medina, M. G. (2018). Avaliação da qualidade da Atenção Pré-natal no Brasil. Saúde em Debate, 42, 111 -126.

Mario, D. N., Rigo, L., Boclin, K. D. L. S., Malvestio, L. M. M., Anziliero, D., Horta, B. L., ... \& Martínez-Mesa, J. (2019). Qualidade do pré-natal no Brasil: Pesquisa Nacional de Saúde 2013. Ciência \& Saúde Coletiva, 24, 1223-1232.

Marques, L. D. S. (2017). Humanização na assistência ao pré-natal e puerpério.

Mendes, R. B., Santos, J. M. D. J., Prado, D. S., Gurgel, R. Q., Bezerra, F. D., \& Gurgel, R. Q. (2020). Avaliação da qualidade do pré-natal a partir das recomendações do Programa de Humanização no Pré-natal e Nascimento. Ciência \& Saúde Coletiva, 25, 793-804.

Modesto, A., \& Livramento, É. A. (2018). A assistência da enfermagem no processo de cuidados humanizados à gestante durante o pré-natal no centro saúde Fonte Inês (Bachelor's thesis, Universidade do Mindelo).

Oliveira, A. S., dos Santos, M. E. P., \& Cavalcante, M. A. B. (2019). A importância do acompanhamento psicológico no ciclo gravídico puerperal. Humanidades \& Inovação, 6(13), 48-54.

Santos, V. L. O. \& Oliveira, E. T. A. C. (2018). Atuação do enfermeiro no período gestacional durante realização do teste rápido anti-hiv

Oliveira, C. S. D., \& Alves, N. N. (2019). Avaliação da assistência ao pré-natal de adolescentes em uma maternidade de referência (Doctoral dissertation)

Oliveira, F. S., Vieira, F., Cecilio, J. O., Guimarães, J. V., \& Campbell, S. H. (2020). A eficácia da educação em saúde na prevenção do trauma mamilar na amamentação: revisão sistemática. Revista Brasileira de Saúde Materno Infantil, 20, 333-345.

Sousa, L. P., Monteiro R. S., do Nascimento, V. B, Neto A. S. S. \& Mendes L. M. C. (2021). Atuação da Equipe de Enfermagem no Teste Rápido Anti - HIV. Rev. enferm UFPE online, 15(1). DOI: 10.5205/1981-8963.2020.244420

Reis, R. S., \& Abi Rached, C. D. (2017). O papel do enfermeiro no acompanhamento de pré-natal de baixo risco utilizando a abordagem centrada na pessoagestante. International Journal of Health Management Review, 3(2).

RIBEIRO, M. S. M. (2020). Assistência pré-natal e a prevenção da morte materna: uma revisão integrativa. 8 - 39.

Sales, B. D. L., Querino, E. T., Sales, J. L. A., \& Carvalho, T. M. (2020). Portfólio acadêmico: assistência da enfermagem na saúde da mulher.

Sardinha, D. M., Maciel, D. O., Gouveia, S. C., Pamplona, F. C., Sardinha, L. M., Carvalho, M. D. S. B. D., \& Silva, A. G. I. D. (2019). Promoção do aleitamento materno na assistência pré-natal pelo enfermeiro. Rev. enferm. UFPE on line, 852-857. https://doi.org/10.5205/1981-8963-v13i03a238361p852$857-2019$

Silva, J. C. B., de Moura Borba, A., da Rocha Santos, A. B., Ferraz, A. C. D., de Amorim Silva, G., da Silva, L. C., ... \& de Carvalho, M. V. G. (2019). aplicação da sistematização da assistência de enfermagem em gestantes atendidas no pré-natal. Revista Ciência Plural, 5(3), 89-102.

Silva, J. D. S. D. (2019). Suplementação de ácido fólico e sulfato ferroso na gestação: estudo qualitativo com gestantes de um município do Recôncavo da Bahia.

Silva, E. P. D., Lima, R. T. D., \& Osório, M. M. (2016). Impacto de estratégias educacionais no pré-natal de baixo risco: revisão sistemática de ensaios clínicos randomizados. Ciência \& Saúde Coletiva, 21, 2935-2948.

Souza, A. L. D. M. D., Zilly, A., Cardelli, A. A. M., Fracarolli, I. F. L., \& Ferrari, R. A. P. (2021). Rede Mãe Paranaense: assistência ao pré-natal entre mulheres nos extremos de idade. Rev. Saúde Pública Paraná (Online), 25-40. DOI10.32811/25954482-2021v4n2p25

Spineli, V. M. C. D. (2019). Conhecimento e autoeficácia em cuidados paliativos dos enfermeiros da Atenção Primária à Saúde (Doctoral dissertation, Universidade de São Paulo). 
Research, Society and Development, v. 10, n. 14, e270101422161, 2021 (CC BY 4.0) | ISSN 2525-3409 | DOI: http://dx.doi.org/10.33448/rsd-v10i14.22161

Strefling, I. D. S. S., Borba, C. B., Demori, C. C., Soares, M. C., Váz, C. H. G. J., \& Santos, C. P. D. (2017). Perceptions of puerperas on nursing care in joint accommodation. Revista de Pesquisa: Cuidado é Fundamental Online, 9(2), 333-339. https://doi.org/10.9789/2175-5361.2017.v9i2.333-339

Viellas, E. F., Domingues, R. M. S. M., Dias, M. A. B., Gama, S. G. N. D., Theme, M. M., Costa, J. V. D., ... \& Leal, M. D. C. (2014). Assistência pré-natal no Brasil. Cadernos de Saúde Pública, 30, S85-S100.

Vilaça, D. M. (2017). Sensibilização da equipe de enfermagem quanto às boas práticas de assistência ao parto na Maternidade Hilda Brandão-Santa Casa de Belo Horizonte.

Zanatta, E., Pereira, C. R. R., \& Alves, A. P. (2017). A experiência da maternidade pela primeira vez: as mudanças vivenciadas no tornar-se mãe. Revista Pesquisas e Práticas Psicossociais, 12(3), 16. 